\title{
Analysis of The Rosenzweig-MacArthur Predator-Prey Model with Anti-Predator Behavior
}

\author{
Ismail Djakaria1, Muhammad Bachtiar Gaib² , Resmawan³ \\ 1,2,3 Department of Mathematics, Universitas Negeri Gorontalo, Indonesia \\ Email: iskar@ung.ac.id,m.tiargaib@gmail.com,resmawan@ung.ac.id
}

\begin{abstract}
This paper discusses the analysis of the Rosenzweig-MacArthur predator-prey model with antipredator behavior. The analysis is started by determining the equilibrium points, existence, and conditions of the stability. Identifying the type of Hopf bifurcation by using the divergence criterion. It has shown that the model has three equilibrium points, i.e., the extinction of population equilibrium point $\left(E_{0}\right)$, the non-predatory equilibrium point $\left(E_{1}\right)$, and the co-existence equilibrium point $\left(E_{2}\right)$. The existence and stability of each equilibrium point can be shown by satisfying several conditions of parameters. The divergence criterion indicates the existence of the supercritical Hopf-bifurcation around the equilibrium point $E_{2}$. Finally, our model's dynamics population is confirmed by our numerical simulations by using the 4th-order Runge-Kutta methods.
\end{abstract}

Keywords: Rosenzweig-MacArthur; predator-prey model; anti-predator behaviour; Hopf Bifurcation; divergence criterion; equilibrium point.

\section{INTRODUCTION}

Population dynamics are the most interesting research in mathematical biology which discusses the interactions that occur between prey and predator in a particular ecosystem [1]. This interaction has implemented to a simple mathematical model known as the Lotka-Volterra predator-prey model [2].

In a mathematical model, the predation process (interaction between prey and predator) is expressed in some form that is known as a functional response. This functional response has classified three functions, i.e. Holling-Type I, Holling-Type II, and Holling-Type III where each type determine the characteristic of the predator [3]. On the progress, Rosenzweig and MacArthur modifying the Lotka-Volterra predator-prey model with the assumption the attack rate of predator increases at a decreasing rate with prey density until it becomes constant due to satiation which is affected by Holling-Type II functional response [4]. Further, some modified of Lotka-Volterra predator-prey model by considering the infectious disease [5]-[7].

Several research has discussed the modification of the Rosenzweig-MacArthur predator-prey model [8] [9] is introduced predator foraging facilitation into Holling-Type II functional response. Furthermore, the Rosenzweig-MacArthur model has modified with various factors, e.g. the stage-structure [10][11], the refuge effect [12][13], the harvesting to one or more population [14][15]. From several studies described above, no one 
considering anti-predator behavior factors.

In this article, the Rosenzweig-MacArthur predator-prey model by [6] modified considering anti-predator behavior factors [16]. These factors can be considered in the model because the dynamics of the model will be very complex when the prey population prefers to defending and provide resistance when the predation process is occurring. The structure of this paper is as follows. In the next section, the methods in our work are described. Then, the analysis of the model has been discussed. Finally, a brief conclusion of our work is given.

\section{METHODS}

The dynamics of the model is analyzed by carrying out the following steps:

1. Modifying the Rosenzweig-MacArthur predator-prey model considering antipredator behavior factors.

2. Simplifying the model by using non-dimensional to reduce the number of parameters and solving the equilibrium points of the model.

3. Identifying the existence, local stability, and global stability of the equilibrium points.

4. Identifying the Hopf-bifurcation type by using the divergence criterion.

5. Demonstrated the numerical simulations of the model to describe the analysis results by using the 4th-order Runge-Kutta method.

\section{RESULTS AND DISCUSSION}

\section{Mathematical Model}

In this article, the mathematical model is formulated based on the following assumptions:

1. The prey population is assumed to grow logistically with an intrinsic growth rate of $r$ and carrying capacity of the environment of $K$ and reduced due to the predation process.

2. The predator population is assumed to grow due to the predation process. $c$ is the conversion rate of the consumed prey into predator births.

3. The predation process follows Holling-Type II functional response which is affected by the encounter rate function where there is foraging facilitation of predator $(w=0), a$ is the saturated rate of the predator, $b$ is coefficient interaction on both population and $h$ is the predator time handling.

4. $m$ is the mortality of predators.

5. $\eta$ is the anti-predator behavior.

From the following assumptions above, the dynamics of the model can be represented by the following set of differential equations:

$$
\begin{aligned}
& \frac{d x}{d t}=r x\left(1-\frac{x}{K}\right)-\frac{(a-b) x y}{y+h(a-b) x} \\
& \frac{d y}{d t}=\frac{c(a-b) x y}{y+h(a-b) x}-m y-\eta x y
\end{aligned}
$$

Where $x$ and $y$ are respectively the densities of prey and predator population at time $t$ and $x(0), y(0)>0$. 
To simplify our analysis, we reduce the number of parameters in system (1) by using the following parameter scales [17]:

$$
x \rightarrow x K, \quad y \rightarrow y(a-b) K h, \quad t \rightarrow \frac{t}{r}
$$

We obtain the following non-dimensional model

$$
\begin{aligned}
& \frac{d x}{d t}=x(1-x)-\frac{\alpha x y}{x+y} \\
& \frac{d y}{d t}=\frac{\beta x y}{x+y}-\gamma y-\delta x y
\end{aligned}
$$

where

$$
\alpha=\frac{(a-b)}{r}, \quad \beta=\frac{c}{h r}, \quad \gamma=\frac{m}{r}, \quad \delta=\frac{\eta K}{r}
$$

\section{Existence and Stability Analysis of Equilibrium Points}

In this section, the equilibrium point of model (2) is obtained by solving [18]:

$$
\begin{aligned}
& x(1-x)-\frac{\alpha x y}{x+y}=0 \\
& \frac{\beta x y}{x+y}-\gamma y-\delta x y=0
\end{aligned}
$$

Thus, from the system ( 3 ), we obtain the following equilibrium points, i.e.:

1. A trivial equilibrium point $E_{0}=(0,0)$, always exists.

2. A non-predator equilibrium point $E_{1}=(1,0)$, always exists too.

3. A co-existence equilibrium point $E_{2}=\left(x^{*}, y^{*}\right)$, where

$$
x^{*}=\frac{\beta-\alpha \beta+\alpha \gamma}{\beta-\alpha \delta}, \quad y^{*}=\frac{(\beta-\alpha \beta+\alpha \gamma)(\beta-\gamma-\delta)}{(\beta-\alpha \delta)(\gamma+\delta-\alpha \delta)}
$$

which exists if

$$
\beta>\alpha(\beta-\gamma), \quad \gamma+\delta<\alpha \delta<\beta
$$

Now, study the local stability of the dynamics of the system (3) around each of equilibrium point. The Jacobian matrix from the system (3) is determined as [19]:

$$
J_{(x, y)}=\left(\begin{array}{cc}
1-2 x-\frac{\alpha y}{x+y}+\frac{\alpha x y}{(x+y)^{2}} & -\frac{\alpha x}{x+y}+\frac{\alpha x y}{(x+y)^{2}} \\
\frac{\beta y}{x+y}-\frac{\beta x y}{(x+y)^{2}}-\delta y & \frac{\beta x}{x+y}-\frac{\beta x y}{(x+y)^{2}}-\gamma-\delta x
\end{array}\right)
$$

By evaluating this Jacobian matrix (4) at each equilibrium point, we obtain the local stability properties of $E_{0}, E_{1}$, and $E_{2}$ as follows. 
Theorem 1. The trivial equilibrium point $E_{0}$ always unstable (saddle).

Proof:

The Jacobian matrix (4) evaluated in equilibrium point $E_{0}$ is given by

$$
J_{\left(E_{0}\right)}=\left(\begin{array}{cc}
1 & 0 \\
0 & -\gamma
\end{array}\right)
$$

So, by solving the characteristic equation, we obtained the eigenvalues of $J_{\left(E_{0}\right)}$ is $\lambda_{1}=1$ and $\lambda_{2}=-\gamma$. It means $\lambda_{1}>0$ and $\lambda_{2}<0$. Therefore, stability of equilibrium point $E_{0}$ is unstable (saddle).

Theorem 2. If $\delta>\beta-\gamma$, then the non-predatory equilibrium point $E_{1}$ of system (2) is locally asymptotically stable.

Proof:

The Jacobian matrix (4) evaluated in equilibrium point $E_{1}$ is given by

$$
J_{\left(E_{1}\right)}=\left(\begin{array}{cc}
-1 & -\alpha \\
0 & \beta-\gamma-\delta
\end{array}\right)
$$

So, by solving the characteristic equation, we obtained the eigenvalues of $J_{\left(E_{1}\right)}$ is $\lambda_{1}=-1$ and $\lambda_{2}=\beta-\gamma-\delta$. It means $\lambda_{1}<0$. Therefore, if $\delta>\beta-\gamma$ then each the eigenvalues of $J_{\left(E_{1}\right)}$ are negatif, and $E_{1}$ is locally asymptotically stable.

Theorem 3. The co-existence equilibrium point $E_{2}$ is locally asymptotically stable if the conditions below are satisfied

\section{Proof:}

$$
\delta^{2}<\frac{\Theta+\Upsilon}{\mathrm{Z}}
$$

The Jacobian matrix (4) evaluated in equilibrium point $E_{1}$ is given by

$$
J_{\left(E_{2}\right)}=\left(\begin{array}{ll}
M_{11} & M_{12} \\
M_{21} & M_{22}
\end{array}\right)
$$

Where

$$
\begin{gathered}
M_{11}=\frac{-\beta^{2}+\alpha \beta^{2}-\alpha \gamma^{2}-2 \alpha \delta(\alpha-1)(\beta-\gamma)-\alpha \delta^{2}+\alpha^{2} \delta^{2}}{(\beta-\alpha \delta)^{2}} \\
M_{12}=-\frac{\alpha(\gamma+\delta-\alpha \delta)^{2}}{(\beta-\alpha \delta)^{2}} \\
M_{21}=\frac{(\beta-\gamma-\delta)\left(\beta^{2} \gamma+\alpha^{2} \gamma \delta^{2}-\beta\left(\gamma^{2}+2 \gamma \delta+\delta^{2}(\alpha-1)^{2}\right)\right)}{(\beta-\alpha \delta)^{2}} \\
M_{22}=-\frac{\beta(\beta-\gamma-\delta)(\gamma+\delta-\alpha \delta)}{(\beta-\alpha \delta)^{2}}
\end{gathered}
$$

By solving the characteristic equation, we obtained the eigenvalues of $J_{\left(E_{2}\right)}$ is

$$
\lambda_{1,2}=\frac{1}{2} \cdot \frac{1}{(\beta-\alpha \delta)^{2}}(A \pm B)
$$


Where

With

$$
A=\mathrm{Z} \delta^{2}-\Theta-\Upsilon \quad \text { and } \quad B=\Psi^{2}-\alpha \Omega
$$

$$
\begin{gathered}
\mathrm{Z}=\left(\alpha^{2}-\alpha+\beta-\alpha \beta\right) \\
\Theta=\delta\left(\beta(\beta-2 \gamma)+2 \alpha^{2}(\beta-\gamma)-\alpha\left(\beta^{2}+2(\beta-\gamma)-\beta \gamma\right)\right) \\
\Upsilon=\beta^{2}(\gamma-\alpha+1)+\gamma^{2}(\alpha+\beta) \\
\Psi=\left(\beta^{2}-\alpha \beta^{2}+\alpha \gamma^{2}+2 \alpha \delta(\alpha-1)(\beta-\gamma)-\alpha \delta^{2}(\alpha-1)-\beta(\beta-\gamma-\delta)(\gamma+\delta-\alpha \delta)\right) \\
\Omega=4(\beta-\gamma-\delta)(\gamma+\delta-\alpha \delta)\left(\beta^{2} \gamma+\alpha^{2} \gamma \delta^{2}-\beta\left((\alpha-1)^{2} \delta^{2}+\gamma^{2}+2 \gamma \delta\right)\right)
\end{gathered}
$$

According to 0 , the stability of equilibrium point $E_{2}$ depending on the value of $A$. If $A<0$, we obtained:

$$
\begin{gathered}
\mathrm{Z} \delta^{2}-\Theta \delta-\Upsilon<0 \\
\mathrm{Z} \delta^{2}<\Theta \delta+\Upsilon \\
\delta^{2}<\frac{\Theta+\Upsilon}{\mathrm{Z}}
\end{gathered}
$$

By the conditions above, the stability of equilibrium point $E_{2}$ is locally asymptotically stable.

Next, study the global stability of the dynamics of the system (3) around equilibrium point $E_{2}$. We obtain the global stability properties of $E_{2}$ by using the Lyapunov function [20] as follows.

Theorem 4. The co-existence equilibrium $E_{2}$ is globally asymptotically stable if the conditions below are satisfied:

$$
x^{*}<\frac{(\alpha-\beta+\gamma+\delta)(\gamma+\delta-\alpha \delta)}{\alpha(\gamma+\delta-\alpha \delta)-(\beta-\gamma-\delta)^{2}}
$$

\section{Proof:}

Define a Lyapunov function as follows

$$
V(x, y)=\left[x-x^{*}-x^{*} \ln \left(\frac{x}{x^{*}}\right)\right]+\left[y-y^{*}-y^{*} \ln \left(\frac{y}{y^{*}}\right)\right]
$$

By using the function $\dot{V}<0, \forall(x, y) \in \mathbb{R}_{2}^{+}$, we obtain:

$$
\begin{gathered}
\frac{\partial V}{\partial x} \cdot \frac{\partial x}{\partial t}+\frac{\partial V}{\partial y} \cdot \frac{\partial y}{\partial t} \leq 0 \\
\left(1-\frac{x^{*}}{x}\right)\left(x(1-x)-\frac{\alpha x y}{x+y}\right)+\left(1-\frac{y^{*}}{y}\right)\left(\frac{\beta x y}{x+y}-\gamma y-\delta x y\right) \leq 0 \\
\left(\frac{(1-x)(x+y)-\alpha y}{x+y}\right)\left(x-x^{*}\right)+\left(\frac{\beta x-\gamma(x+y)-\delta x(x+y)}{x+y}\right)\left(y-y^{*}\right) \leq 0
\end{gathered}
$$

For $(x, y) \in \mathbb{R}_{2}^{+}$, we obtain: 


$$
\begin{gathered}
-\alpha+\alpha x^{*}+\beta-\gamma-\delta-(\beta-\gamma-\delta) y^{*}<0 \\
-\alpha+\alpha x^{*}+\beta-\gamma-\delta-x^{*} \frac{(\beta-\gamma-\delta)^{2}}{(\gamma+\delta-\alpha \delta)}<0 \\
x^{*}\left(\frac{\alpha(\gamma+\delta-\alpha \delta)-\left((\beta-\gamma-\delta)^{2}\right)}{(\gamma+\delta-\alpha \delta)}\right)<\alpha-\beta+\gamma+\delta \\
x^{*}<\frac{(\gamma+\delta-\alpha \delta)(\alpha-\beta+\gamma+\delta)}{\alpha(\gamma+\delta-\alpha \delta)-\left((\beta-\gamma-\delta)^{2}\right)}
\end{gathered}
$$

By the conditions above, the stability of equilibrium point $E_{2}$ is globally asymptotically stable.

\section{Analysis of Hopf Bifurcation Type}

In this section, we'll define the Hopf-bifurcation type by using the divergence criterion [21]. System (3) underwent a Hopf-bifurcation when it satisfies the following conditions:

$$
\delta^{2}<\frac{\Theta+\Upsilon}{\mathrm{Z}} \quad \text { and } \quad \alpha>\frac{\Psi^{2}}{\Omega}
$$

To determine the Hopf-bifurcation type of system (3) on equilibrium point $E_{2}$, then we formed a new system. Let $\phi(x, y)$ is a divergence of $(a f, a g)$. We obtain the coefficient value of $a(x, y)$ of the system (3) when the parameter value $\alpha=2, \beta=0.79, \gamma=0.5$, and $\delta=0.0186$ with equilibrium point $E_{2}^{*}=(0.279 ; 0.157)$ as follows:

$$
a(x, y)=1+6.956 x+13,386 y-6.77 x^{2}+32.968 x y+55.507 y^{2}
$$

So that a new system is obtained:

$$
\begin{gathered}
z(x, y)=\left(1+6.956 x+13,386 y-6.77 x^{2}+32.968 x y+55.507 y^{2}\right) \\
\left(x(1-x)-\frac{\alpha x y}{x+y}\right) \\
w(x, y)=\left(1+6.956 x+13,386 y-6.77 x^{2}+32.968 x y+55.507 y^{2}\right) \\
\left(\frac{\beta x y}{x+y}-\gamma y-\delta x y\right)
\end{gathered}
$$

By linearizing system (4), we obtained:

$$
J_{\left(E_{2}^{*}\right)}=\left(\begin{array}{ll}
1.337 & -6.002 \\
0.732 & -1.337
\end{array}\right)
$$

By solving the characteristic equation, we obtained the eigenvalues of $J_{\left(E_{2}^{*}\right)}$ is

$$
\lambda_{1,2}= \pm 1.615 i
$$

For a system (5) to obtain the eigenvalues of conjugate complex numbers, then we can analyze the Hopf-bifurcation of system (3) type by looking at the divergence value of system (3). We obtained:

$$
\phi_{x x}\left(E_{2}^{*}\right)=-21.109
$$


Based on the divergence value above, a stable limit cycle appears in the system (3). Therefore, system (3) underwent a Supercritical Hopf-bifurcation.

\section{Numerical Simulations}

In this section, the numerical simulation is solved using the 4th-order Runge-Kutta method [22] with initial conditions and some values of the parameters. We choose the following set of parameter values:

$$
\alpha=2, \quad \beta=0.79, \quad \gamma=0.5
$$

With different parameter control values as follows $\delta_{1}=0.011, \delta_{2}=0.0186$ and $\delta_{3}=$ 0.026. We using the initial condition is $x(0)=0.3$ and $y(0)=0.3$.

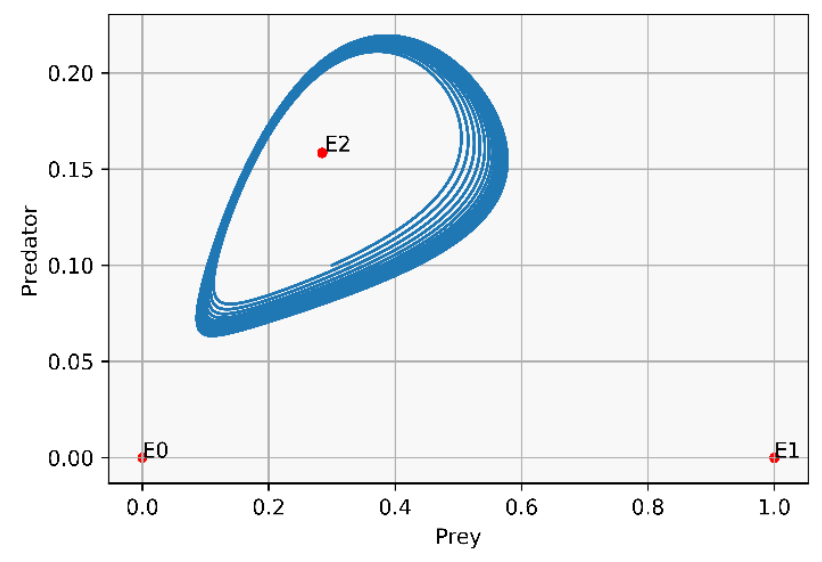

(a)

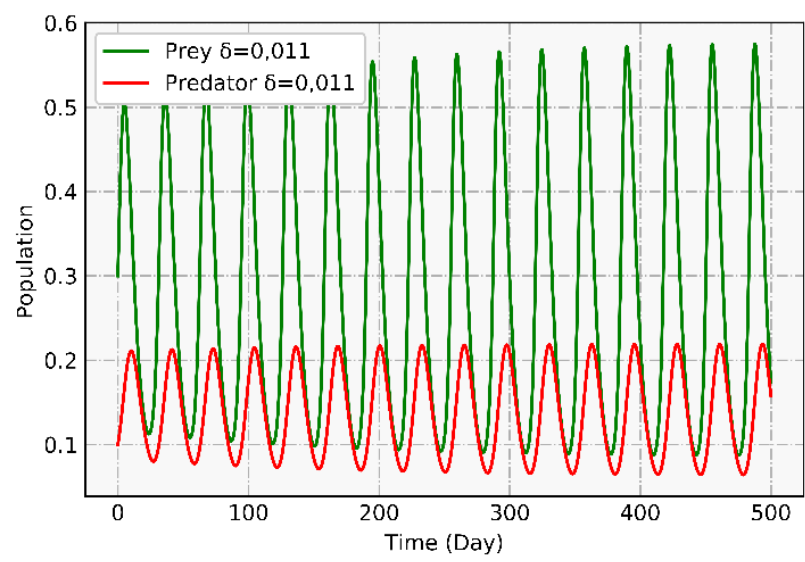

(b)

Figure 1. (a) Phase Portrait of Case 1 and (b) Time-Series Portrait

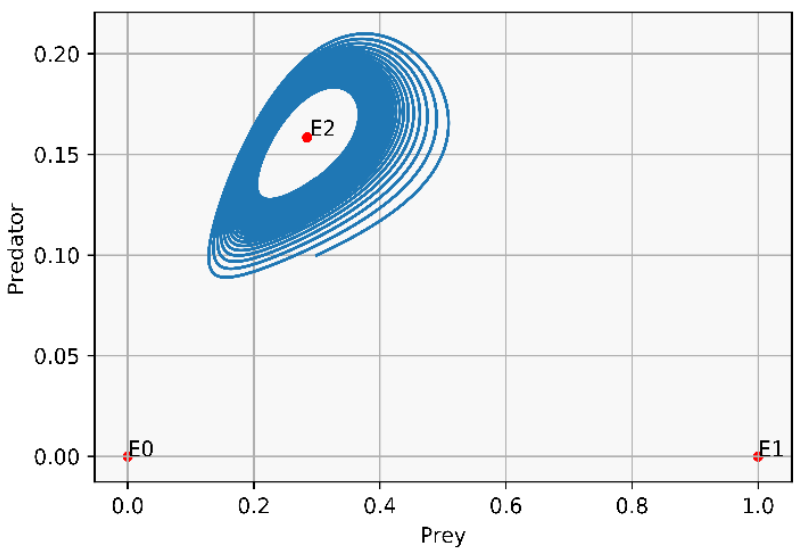

(a)

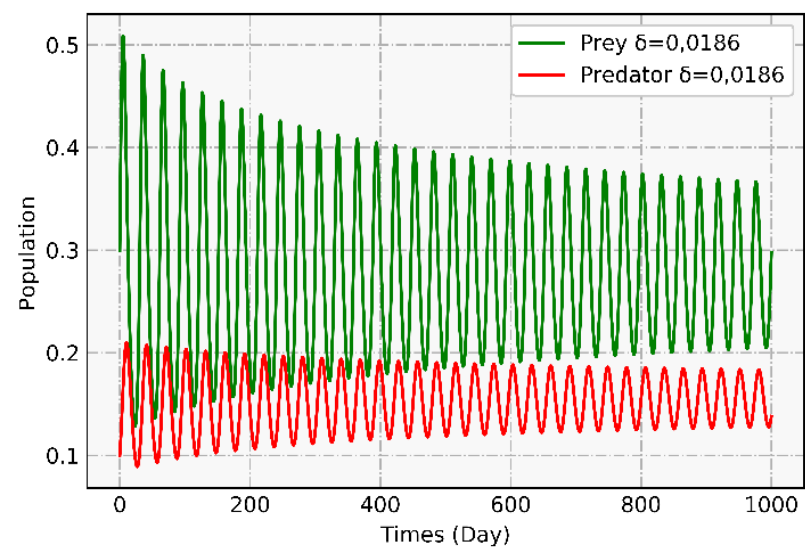

(b)

Figure 2. (a) Phase Portrait of Case 2 and (b) Time-Series Portrait

In case 1, we obtained the dynamics of the solution on the system (3) with parameter control values $\delta_{1}=0.011$. Based on figure

1(a), the trivial equilibrium point $E_{0}=(0,0)$ is unstable (saddle) with eigenvalues $\lambda_{1}=1$ and $\lambda_{2}=-0.5$. This coincides with Theorem 1 . The non-predator equilibrium point $E_{1}=(1,0)$ is unstable (saddle) with eigenvalues $\lambda_{1}=-1$ and $\lambda_{2}=0.279$. This coincides with Theorem 2 on condition $\delta<\beta-\gamma$. The co-existence equilibrium point $E_{2}=(0.273 ; 0.156)$ is unstable (spiral) with eigenvalues $\lambda_{1,2}=0.003 \pm 0.220 i$. This 
coincides with Theorem 3 on condition $\delta^{2}<\frac{\Theta+\Upsilon}{\mathrm{Z}}$. Based on figure 1(b), the prey population and predator population have increased and decreased of total populations. The case continuously oscillates with a greater deviation value. Hence, both population is unstable to a specific point.

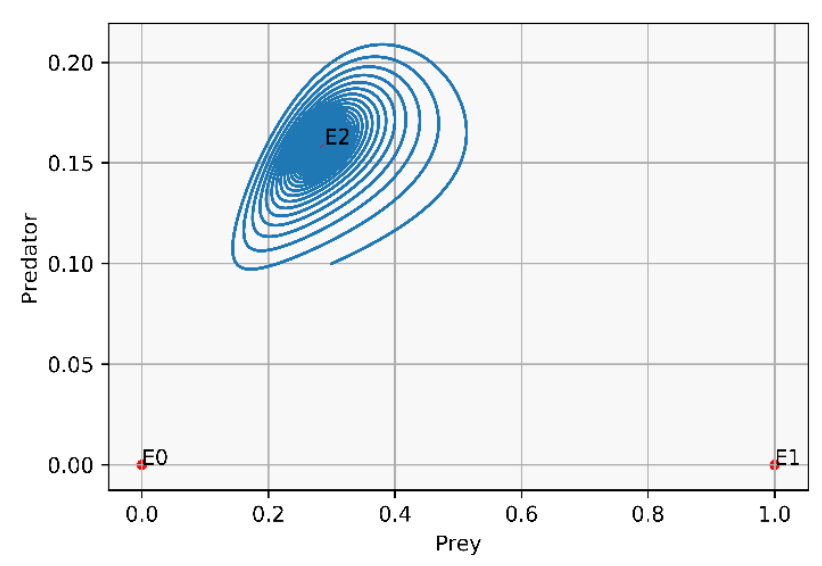

(a)

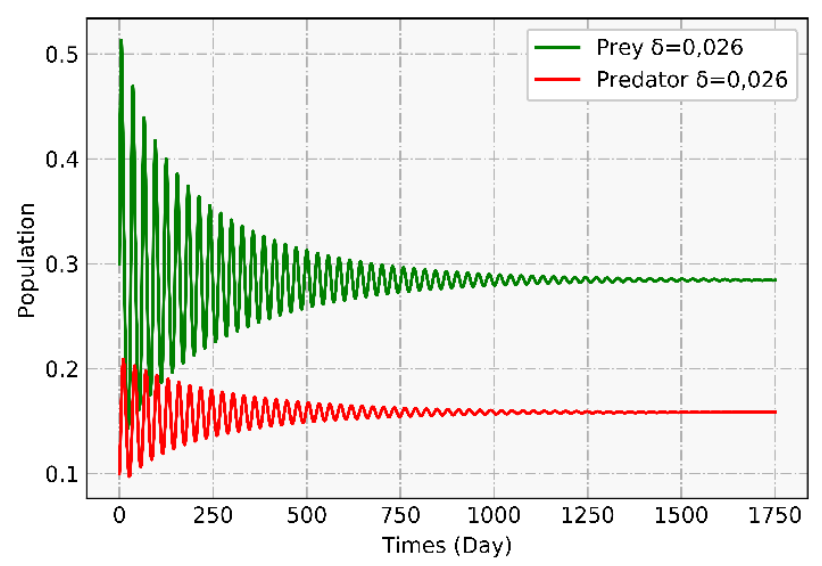

(b)

Figure 3. (a) Phase Portrait of Case 3 and (b) Time-Series Portrait

In case 2, we obtained the dynamics of the solution on the system (3) with parameter control values $\delta_{1}=0.0186$. Based on figure $2(\mathbf{a})$, the trivial equilibrium point $E_{0}=(0,0)$ is unstable (saddle) with eigenvalues $\lambda_{1}=1$ and $\lambda_{2}=-0.5$. This coincides with Theorem 1. The non-predator equilibrium point $E_{1}=(1,0)$ is unstable (saddle) with eigenvalues $\lambda_{1}=-1$ and $\lambda_{2}=0.271$. This coincides with Theorem 2 on condition $\delta<\beta-\gamma$. The coexistence equilibrium point $E_{2}=(0.279 ; 0.157)$ is center (spiral) with eigenvalues $\lambda_{1,2}=$ $\pm 0.220 i$. This coincides with Theorem 3 on condition $\delta^{2}=\frac{\Theta+\Upsilon}{\mathrm{Z}}$. Based on figure 2(b), the oscillations that occur have a smaller deviation value. This condition explains that there is a stability transition from unstable to stable to a specific point. This stability transition has led to the appearance of Hopf-bifurcation.

In case 3, we obtained the dynamics of the solution on the system (3) with parameter control values $\delta_{1}=0.026$. Based on figure 3(a), the trivial equilibrium point $E_{0}=(0,0)$ is unstable (saddle) with eigenvalues $\lambda_{1}=1$ and $\lambda_{2}=-0.5$. This coincides with Theorem 1. The non-predator equilibrium point $E_{1}=(1,0)$ is unstable (saddle) with eigenvalues $\lambda_{1}=-1$ and $\lambda_{2}=0.264$. This coincides with Theorem 2 on condition $\delta<\beta-\gamma$. The coexistence equilibrium point $E_{2}=(0.285 ; 0.159)$ is stable (spiral) with eigenvalues $\lambda_{1,2}=$ $-0.003 \pm 0.220 i$. This coincides with Theorem 3 on condition $\delta^{2}>\frac{\Theta+\Upsilon}{Z}$. Based on figure 3(b), the dynamics between prey and predator begin to stabilize at 1500 days to a specific point.

\section{CONCLUSIONS}

The Rosenzweig-MacArthur predator-prey model with anti-predator behavior has been studied. From the analysis of system (2), we obtain three equilibrium points, i.e., the trivial equilibrium point $\left(E_{0}\right)$, the non-predatory equilibrium point $\left(E_{1}\right)$, and the coexistence equilibrium point $\left(E_{2}\right)$. The local stability conditions of each equilibrium point have been appointed, and the global stability conditions of the co-existence equilibrium 
point $\left(E_{2}\right)$ have been obtained. Our analysis also showed that the model occurs a Supercritical Hopf-bifurcation by using the divergence criterion. Numerical analytic has been simulated to verify the theoretical results. No one extinction matters in any population.

\section{REFERENCES}

[1] P. B. Turchin, Complex Population Dynamics: A Theoretical/Empirical Synthesis, Priceton University Press, 2003.

[2] J. D. Murray, Mathematical Biology: An Introduction, 3rd Edition, Springer-Verlag, 2002.

[3] C. S. Holling, "Some Characteristic of Simple Types of Predation and Parasitism", The Canadian Entomologist, vol. 91, no. 7, pp. 385-398, 1959.

[4] M. L. Rosenzweig and R. H. MacArthur, "Graphical Representation and Stability Conditions of Predator-Prey Interactions", The American Naturalist, vol. 895, pp. 209-223, 1963.

[5] N. Hasan, R. Resmawan, and E. Rahmi, "Analisis Kestabilan Model Eko-Epidemiologi dengan Pemanenan Konstan pada Predator," J. Mat. Stat. dan Komputasi, vol. 16, no. 2, pp. 121-142, Dec. 2020.

[6] S. H. Arsyad, R. Resmawan, and N. Achmad, "Analisis Model Predator-Prey LeslieGower dengan Pemberian Racun pada Predator," J. Ris. dan Apl. Mat., vol. 4, no. 1, pp. 1-16, 2020.

[7] S. Maisaroh, R. Resmawan, and E. Rahmi, "Analisis Kestabilan Model Predator-Prey dengan Infeksi Penyakit pada Prey dan Pemanenan Proporsional pada Predator," Jambura J. Biomath, vol. 1, no. 1, pp. 8-15, 2020.

[8] L. Berec, "Impacts of Foraging Facilitation Among Predators on Predator-Prey Dynamics", Bulletin of Mathematical Biology, vol. 72, pp. 94-121, 2010.

[9] L. Pribylova and A. Peniaskova, "Foraging Facilitation Among Predators and Its Impact on The Stability of Predator-Prey Dynamics", Ecological Complexity, vol. 29, pp. 30-39, 2017.

[10] M. Moustafa, M. H. Mohd, A. I. Ismail, and F. A. Abdullah, "Stage Structure and Refuge Effects in The Dynamical Analysis of a Fractional-Order Rosenzweig-MacArthur Prey-Predator Model", Progress in Fractional Differentiation and Application, vol. 5, no. 1, pp. 49-64, 2019.

[11] L. K. Beay and M. Saija, "A Stage-Structure Rosenzweig-MacArthur Model with Effect of Prey Refuge", Jambura Journal of Biomathematics, vol. 1, no. 1, pp. 1-7, 2020.

[12] E. Alamanza-Vasquez, R. D. Ortiz-Ortiz, and A. M. Marin-Ramirez, "Bifurcations in The Dynamics of Rosenzweig-MacArthur Predator-Prey Model Considering Saturated Refuge for The Preys", Applied Mathematical Sciences, vol. 9, pp. 74757482, 2015.

[13] M. Moustafa, M. H. Mohd, A. I. Ismail, and F. A. Abdullah, "Dynamical Analysis of a Fractional-Order Rosenzweig-MacArthur Model Incorporating a Prey Refuge", Chaos, Solitons and Fractals, vol. 109, pp. 1-13, 2018. 
[14] A. Suryanto, I. Darti, H. S. Panigoro, and A. Kilicman, "A Fractional-Order PredatorPrey Model with Ratio-Dependent Functional Response and Linear Harvesting", Mathematics, vol. 7, no. 11, pp. 1-13, 2019.

[15] H. S. Panigoro, A. Suryanto, W. M. Kusumawinahyu, and I. Darti, "A RosenzweigMacArthur Model with Continuous Threshold Harvesting in Predator Involving Fractional Derivatives with Power Law and Mittag-Leffler Kernel", Axioms, vol. 9, no. 122 pp. 1-23, 2020.

[16] S. G. Mortoja, P. Panja, and S. K. Mondal, "Dynamics of a Predator-Prey Model with Stage-Structure on Both Species and Anti-Predator Behavior", Informatics in Medicine Unlocked, vol. 10, pp. 50-57, 2018.

[17] S. H. Strogatz, Nonlinear Dynamics and Chaos with Application to Physics, Biology Chemistry and Engineering, West-View Press, 2015.

[18] L. Perko, Differential Equations and Dynamical Systems, 3rd Edition, SpringerVerlag, 2001.

[19] J. K. Hale and H. Kocak, Dynamic and Bifurcation, Springer-Verlaag, 1991.

[20] R. Sundari and E. Apriliani, "Konstruksi Fungsi Lyapunov untuk Menentukan Kestabilan", Jurnal Sains dan Seni ITS, vol. 6, no. 1, pp. 28-32, 2017.

[21] S. S. Pilyugin and P. Waltman, "Divergence Criterion for Generic Planar System", SIAM J Appl. Math., vol. 64, pp. 81-93, 2003.

[22] A. Suryanto, Metode Numerik untuk Persamaan Diferensial Biasa dan Aplikasinya dengan Matlab, Universitas Negeri Malang, 2017. 